ARTICLE

https://doi.org/10.1038/s41467-019-14192-0

\title{
Substrate induced nanoscale resistance variation in epitaxial graphene
}

\author{
Anna Sinterhauf (1) ${ }^{1 \star}$, Georg A. Traeger (10) ${ }^{1}$, Davood Momeni Pakdehi², Philip Schädlich (1) 3,4, Philip Willke (1) 5,6, \\ Florian Speck ${ }^{3,4}$, Thomas Seyller (10 ${ }^{3,4}$, Christoph Tegenkamp (10 ${ }^{3}$, Klaus Pierz (1) ${ }^{2}$, Hans Werner Schumacher ${ }^{2}$ \& \\ Martin Wenderoth (1D ${ }^{1 \star}$
}

Graphene, the first true two-dimensional material, still reveals the most remarkable transport properties among the growing class of two-dimensional materials. Although many studies have investigated fundamental scattering processes, the surprisingly large variation in the experimentally determined resistances is still an open issue. Here, we quantitatively investigate local transport properties of graphene prepared by polymer assisted sublimation growth using scanning tunneling potentiometry. These samples exhibit a spatially homogeneous current density, which allows to analyze variations in the local electrochemical potential with high precision. We utilize this possibility by examining the local sheet resistance finding a significant variation of up to $270 \%$ at low temperatures. We identify a correlation of the sheet resistance with the stacking sequence of the $6 \mathrm{H}$ silicon carbide substrate and with the distance between the graphene and the substrate. Our results experimentally quantify the impact of the graphene-substrate interaction on the local transport properties of graphene.

\footnotetext{
${ }^{1}$ IV. Physikalisches Institut, Georg-August-Universität Göttingen, Friedrich-Hund-Platz 1, 37077 Göttingen, Germany. ${ }^{2}$ Physikalisch-Technische Bundesanstalt, Bundesallee 100, 38116 Braunschweig, Germany. ${ }^{3}$ Institut für Physik, Technische Universität Chemnitz, Reichenhainer Straße 70,09126 Chemnitz, Germany. ${ }^{4}$ Zentrum für Materialien, Architekturen und Integration von Nanomembranen (MAIN), Technische Universität Chemnitz, Rosenbergstraße 6, 09126 Chemnitz, Germany. ${ }^{5}$ Center for Quantum Nanoscience, Institute for Basic Science (IBS), Seoul 03760, Republic of Korea. ${ }^{6}$ Ewha Womans University, Seoul 03760, Republic of Korea. *email: anna.sinterhauf@uni-goettingen.de; martin.wenderoth@uni-goettingen.de
} 
C harge transport in epitaxial graphene has been subject of theoretical and experimental investigation since its first electronic characterization ${ }^{1}$. The high quality and its $2 \mathrm{D}$ nature make epitaxial graphene the perfect system to study fundamental transport properties on the nanometer scale. Consequently, in a series of experiments-based on scanning tunneling potentiometry (STP $)^{2}$ or four-point-probe micro$\mathrm{scopy}^{3}$ - several groups have focused on local properties like the sheet resistance and the impact of scattering centers like single substrate steps ${ }^{4,5}$ or the transition from monolayer to bilayer graphene on transport ${ }^{6,7}$. From these results, it is qualitatively well understood that the transport properties of epitaxial graphene are not homogeneous on the nanometer scale. Substrate steps or monolayer-bilayer junctions act as local scattering centers. In addition, for epitaxial graphene on $\mathrm{SiC}(0001)$ it is well known that interaction with the substrate drastically affects graphene's transport properties. In order to reduce this inherent proximity effect, i.e., to improve the transport properties of the graphene sheet, different strategies were pursued such as the refinement of the growth process ${ }^{8-10}$, the use of suitable dielectric substrates like boron nitride ${ }^{11}$, the decoupling of the substrate by intercalation ${ }^{12}$, or the preparation of suspended graphene $^{13}$. Moreover, the proximity effect can be deliberately exploited to specifically tune the properties of a graphene sheet $^{14-18}$. For example, the almost negligible spin-orbit coupling can be significantly increased by bringing the graphene layer into contact with transition metal dichalcogenides ${ }^{14,15}$ and proximity superconductivity can be observed in graphene in the vicinity of superconducting materials ${ }^{19}$.

In the context of charge transport in epitaxial graphene, a locally varying potential landscape and a spatially inhomogeneous current density are induced by local defects like substrate steps and local variations of the coupling between the graphene layer and the substrate. Analyzing the published results for resistances assigned to specific defects in epitaxial graphene, one finds a large spread $^{4-7,20-25}$. The strong variation in the experimental values of sheet or defect resistances determined by local probe measurements is likely due to the lack of information about the actual local current density. Replacing the probe by a single-electron transistor allows simultaneous measurement of local voltage drop and current distribution in $2 \mathrm{D}$ materials ${ }^{26}$ with a lateral resolution in the range of $350 \mathrm{~nm}^{26}$. In comparison, STP has an angstrom resolution ${ }^{27}$ and measures the local electrochemical potential with high accuracy, but local variations in the current density are experimentally not accessible and are indistinguishable from spatial variations of the sheet resistance. For conventionally grown graphene on $\mathrm{SiC}(0001)$, typically monolayers as well as bilayers are present. Monolayer-bilayer transitions represent strong scattering centers and cause a significant variation of the local current density. Having no better approach, so far local transport properties have been determined using an averaged (sometimes even macroscopic) current density for the whole sheet.

In this study, we show that the high quality of epitaxial monolayer graphene samples grown by polymer assisted sublimation growth (PASG) opens a promising way to quantify also delicate local transport properties with high precision. Applying the PASG method, it is possible to grow large-scale monolayer graphene sheets without bilayer formation ${ }^{9,28}$ on $\mathrm{SiC}$ substrates with ultra-low step heights. This allows for local transport investigations of monolayer graphene on terraces with different $\mathrm{SiC}$ terminations free from bilayer and step edge effects.

The aim of this work is to test for local variations in the sheet resistance of epitaxial graphene and to unravel possible intrinsic proximity effects induced by the presence of the substrate.

\section{Results}

Homogeneity of the current density. The local electric field as well as the local current density are needed to determine the local sheet resistance. While STP measures the local voltage drop, the local current density is a priori unknown. In STP studies, it is replaced by an averaged value, e.g., given by the total current and the geometry of the sample. While this approximation has severe limits for locally inhomogeneous samples, the excellent lateral homogeneity of the PASG graphene parallel to the steps, the absence of bilayer graphene and the low impact of steps on the overall resistance ${ }^{28}$, drastically reduce lateral current density variations $^{29}$. In our STP setup, the current flow was deliberately driven parallel to the miscut of the SiC sample, resulting in an overall voltage drop perpendicular to the substrate steps ([1]00] direction). The experimental geometry and the assumption that graphene terraces have a constant sheet resistance parallel to the steps result in a constant average current density on all terraces. To estimate the remaining variation in $j_{\text {local }}(x, y)$, we have modeled the local current density for a given surface geometry (Fig. 1a) taken from constant current topographies (CCT) with a resistor network ${ }^{6,22,30}$ and find that the resulting current density exhibits a maximum variation of up to $7 \%$ (Fig. 1 b). By carefully selecting regions away from complex step configurations, e.g., convergence of two steps, the current density can be considered as highly homogeneous (compare Supplementary Table 1). It is $j_{\text {local }}(x, y) \approx j_{\text {local }}=(0.89 \pm 0.01) \mathrm{Am}^{-1}$ for an applied voltage along the graphene layer perpendicular to the substrate steps of $1 \mathrm{~V}$ at $T=300 \mathrm{~K}$. Comparing the lateral variation of the current density in PASG graphene samples with conventionally grown epitaxial graphene, it becomes obvious that local variations in

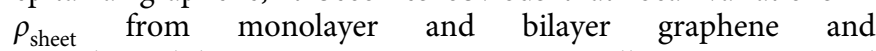
monolayer-bilayer junctions in conventionally grown epitaxial graphene induce a strong variation of $j_{\text {local }}(x, y)$ (Supplementary Fig. 1).

Local variation of $\rho_{\text {sheet }}$ at temperature $T=300 \mathrm{~K}$. Large scale constant current topographies (Fig. 1a) reveal a surface with single, double as well as triple substrate steps and no bilayer regions as expected for epitaxial graphene grown by $\mathrm{PASG}^{9,10,28}$ STP measurements investigating $\rho_{\text {sheet }}$ are performed across all step configurations in Fig. 1a, the corresponding voltage drops $V_{\text {STP }}$ are shown in Fig. 1d-f. Interestingly, to the left and to the right of single substrate steps we find a different gradient of $V_{\text {STP }}$ (Fig. 1d), indicated by the dashed blue and red lines representing the slope to the left and to the right of the step, respectively. Since the current density is constant, this directly proves that the top and bottom terrace have different sheet resistances. This finding also holds for terraces connected by a double substrate step (Fig. 1e), whereas the identical $\rho_{\text {sheet }}$ is measured when crossing a triple substrate step (Fig. 1f). For all step configurations, an additional voltage drop at the topographic position of the step is observed, which is commonly explained by a potential barrier induced by the step due to detachment of the graphene sheet from the substrate $4,5,31$.

In order to further investigate spatial variations of $\rho_{\text {sheet }}$, we have measured large sequences of steps. The topographic analysis has shown that instead of a random distribution of step heights, a well-defined sequence of the step heights shows up: along the [1100] direction, either a triple substrate step is present or a single substrate step and a double substrate step are observed. These characteristic step patterns for PASG graphene on $6 \mathrm{H}-\mathrm{SiC}$ have recently been reported in an Atomic Force Microscopy (AFM) study and have been attributed to the growth process ${ }^{28}$. The detailed STP analysis of large sequences of substrate steps allows deducing two implications: firstly, the evaluation shows that at 
a

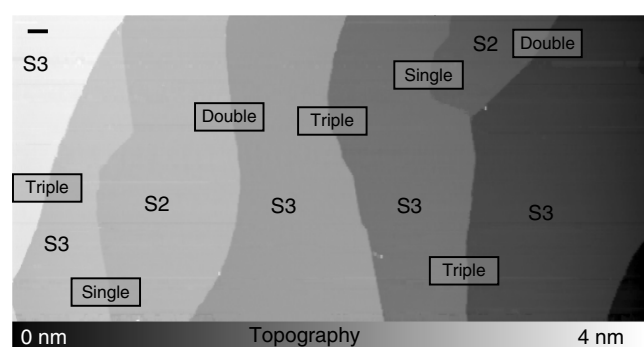

b

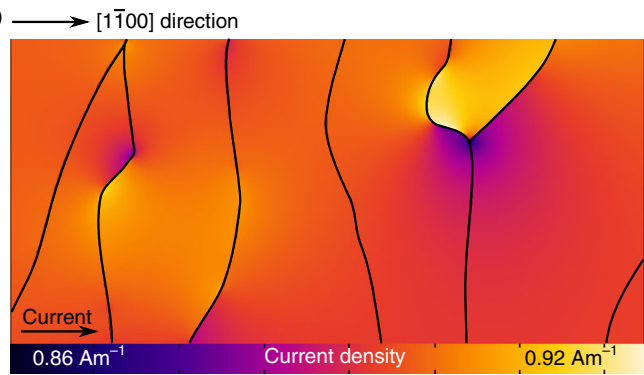

C

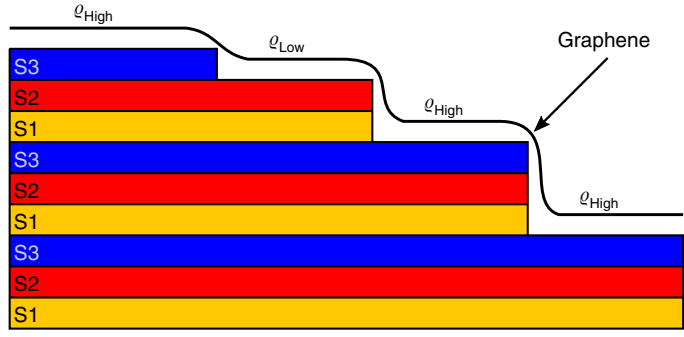

d

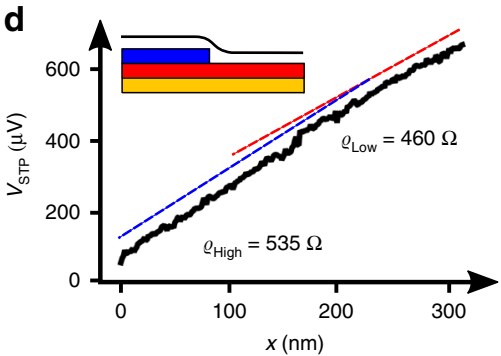

e

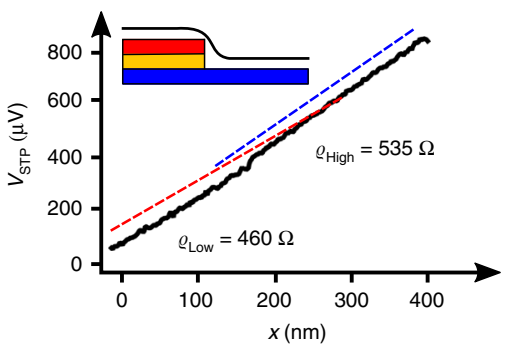

f



Fig. 1 Current density and evaluation of the local sheet resistance at room temperature. a Large-scale constant current topography $(2 \mu \mathrm{m} \times 1 \mu \mathrm{m}$, $V_{\text {Bias }}=0.5 \mathrm{~V}, I_{\mathrm{T}}=0.03 \mathrm{nA}$ ). S1, S2, and S3 indicate the fundamental bilayers (and thus the stacking) of the SiC substrate, details are given in the discussion. STP measurements were performed in the marked areas (black boxes). The height of the steps is denoted in the marked areas, the scale bar is $100 \mathrm{~nm}$. b Using the macroscopic ohmic resistance, the sample geometry shown in a and step resistances of $6 \Omega \mu \mathrm{m}, 12 \Omega \mu \mathrm{m}, 18 \Omega \mu \mathrm{m}$ for single, double, and triple steps, respectively, the [1100] component of the local current density $j_{\text {local }}(x, y)$ is calculated with a finite element simulation. c Schematic side view of the crystal structure of $6 \mathrm{H}-\mathrm{SiC}$. $\mathbf{d}$ Measured voltage drop along the graphene layer induced by the cross voltage $V_{\text {cross }}$ when crossing a single step. Dashed lines represent the slope of the voltage drop (shifted for clarity). e Corresponding measurement for a double step and f for a triple step.

$300 \mathrm{~K}$ the sheet resistance across a given terrace is constant (Supplementary Fig. 2). Secondly, from STP measurements on more than 40 terraces, we extract two clearly distinct sheet resistances, which we refer to as $\rho_{\text {High }}$ and $\rho_{\text {Low }}$. The mean $\rho_{\text {High }}$ is $535 \Omega$ and the mean $\rho_{\text {Low }}$ is $460 \Omega$. The mean $\rho_{\text {High }}$ and $\rho_{\text {Low }}$ deviate by $(14 \pm 1) \%$ from each other at room temperature. Moreover, $\rho_{\text {High }}$ as well as $\rho_{\text {Low }}$ show a variation from terrace to terrace of $\pm 20 \Omega$.

Temperature-dependence of $\rho_{\text {sheet }}$. In order to disentangle possible scattering processes and to understand the difference between $\rho_{\text {High }}$ and $\rho_{\text {Low }}$, we performed further temperaturedependent STP measurements at $77 \mathrm{~K}$ and $8 \mathrm{~K}$ (Fig. 2a). In this study two different samples, both prepared using the PASG method, from two different batches were analyzed as summarized in Fig. 2a. Both samples show good quantitative and qualitative agreement at room temperature, low-temperature measurements were carried out on one of the two samples. This allows on the one hand to compare results for different samples and on the other hand to discuss the temperature dependence of the sheet resistance for one given sample.

We find an overall decrease in the sheet resistance with decreasing temperature, which is supported by macroscopic transport measurements in four-point van der Pauw geometry
(Supplementary Fig. 3) and in agreement with published results $^{32,33}$. The relative reduction in $\rho_{\text {High }}$ with decreasing temperature is slightly smaller, i.e., it reduces by $32 \%$, from $535 \Omega$ to approximately $365 \Omega$ at $8 \mathrm{~K}$, compared with the temperature-dependence of $\rho_{\text {Low }}$, which declines from $460 \Omega$ to an average value of $250 \Omega$ at $8 \mathrm{~K}$, i.e., it reduces by $45 \%$. Besides the overall reduction of $\rho_{\text {sheet }}$, a surprising large increase in the spread in the data is observed with decreasing temperature. At $8 \mathrm{~K}$, a maximum variation in $\rho_{\text {sheet }}$ of $\sim 270 \%$ between the lowest value for $\rho_{\text {Low }}$ and the highest value for $\rho_{\text {High }}$ is observed. On adjacent terraces a maximum variation of $178 \%$ (Supplementary Fig. 4 ) is measured. In the following, we will use $\Delta_{\text {rel }}=\left(\rho_{\text {sheet1 }}-\right.$ $\left.\rho_{\text {sheet } 2}\right) /\left(\rho_{\text {sheet } 1}+\rho_{\text {sheet } 2}\right) / 2$ to quantify the relative change in the sheet resistance for adjacent terraces (Fig. 2b). Regardless of the temperature, when crossing a triple substrate step, the variation in $\rho_{\text {sheet }}$ is small, i.e., $\Delta_{\text {rel }}<3 \%$. In contrast to this, the relative variation in $\rho_{\text {sheet }}$ to the left and to the right of single and double substrate steps increases when going from $300 \mathrm{~K}$ to $77 \mathrm{~K}$. In particular, for terraces connected by single or double substrate steps a mean relative change of more than $30 \%$ is measured. In both cases $\Delta_{\text {rel }}$ slightly decreases from $77 \mathrm{~K}$ to $8 \mathrm{~K}$.

Analysis of the surface morphology of steps and terraces. To further investigate the local variation of the transport properties, 

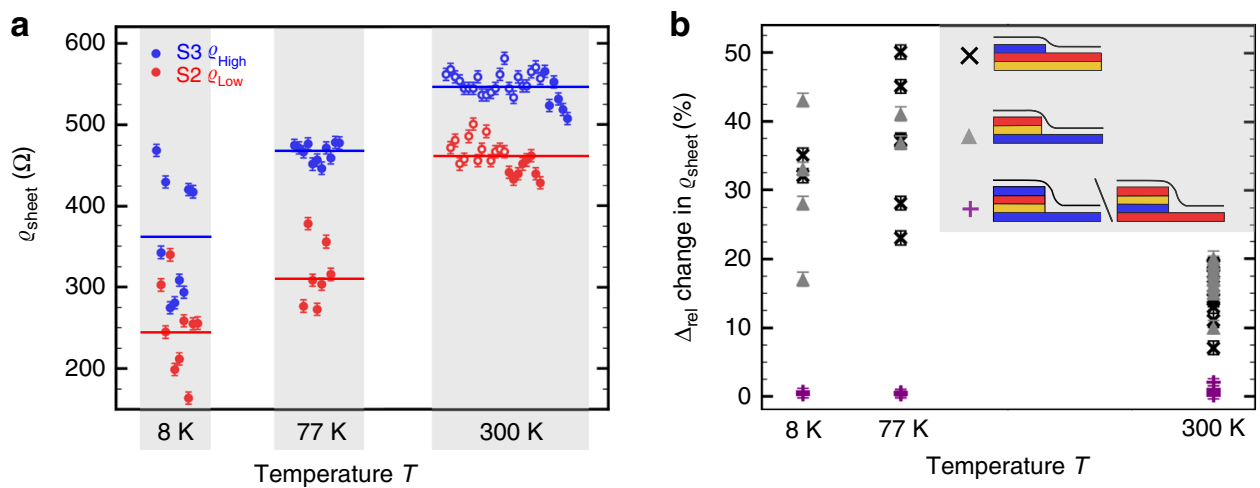

Fig. 2 Temperature-dependence of the sheet resistance. a Sheet resistance at $8 \mathrm{~K}, 77 \mathrm{~K}$, and $300 \mathrm{~K}$ acquired on more than 80 terraces for two samples (indicated by open and filled symbols), solid horizontal lines indicate the mean value for a given terrace and temperature. $\mathbf{b}$ Change in sheet resistance for adjacent terraces for all three cases $\mathrm{S} 3 \rightarrow \mathrm{S} 2, \mathrm{~S} 2 \rightarrow \mathrm{S} 3$, and $\mathrm{S} 3 \rightarrow \mathrm{S} 3 / \mathrm{S} 2 \rightarrow \mathrm{S} 2$ as a function of temperature. Error bars indicate the experimental measurement uncertainty of the individual data points. Source data are provided as a Source Data file.


Fig. 3 Analysis of the step height of single and double steps. a Line profile through a constant current topography showing adjacent terraces S2, S3, S2, connected by a single substrate step followed by a double substrate step recorded at 77K. The line profile reveals a deviation from the step heights of the $\mathrm{SiC}$ substrate steps. $\mathbf{b}$ Schematic representation of the correlation between step height and sheet resistance illustrating a locally varying distance $d$ between the graphene layer and the substrate. c Difference in the sheet resistance for adjacent terraces for single and double steps measured at 8K as a function of the deviation of the step height. Details are given in the "Discussion". Error bars indicate the experimental measurement uncertainty of the individual data points. Source data are provided as a Source Data file.

structural and electronic properties of PASG graphene have been analyzed on different length scales on the same samples. On a mesoscopic scale the surface is characterized by single, double and triple steps, resulting from the miscut of the SiC substrate. Surprisingly, we rarely found the expected height of the substrate steps, i.e., multiple of $0.25 \mathrm{~nm}^{34}$. Instead, we observed deviations of the step height with smaller as well as larger values for both single and double steps. As an example, Fig. 3a displays a line profile across a step sequence consisting of a single substrate step and a double substrate step.

For this specific step configuration, the analysis reveals a step height $>0.25 \mathrm{~nm}$ for the single substrate step and a step height $<0.5 \mathrm{~nm}$ for the double substrate step, i.e., also the combined step height does not fit to the expected value of three times $0.25 \mathrm{~nm}$. Assuming that different step heights correspond to different distances between the graphene monolayer and the substrate, step sequences (Fig. 3) allow to study the relation between distance and sheet resistance. As usual for graphene on $\mathrm{SiC}$, also for PASG graphene a buffer layer forms between the $\mathrm{SiC}$ surface and the graphene sheet ${ }^{9}$. However, since we cannot pin down the vertical position of the height variation, we use the wording 'distance to the substrate'.

The corresponding STP measurement reveals a higher conductivity on terrace III compared with terrace I (see Fig. 3b), indicating that a larger distance results in a smaller resistance. Details on the dependence of $\rho_{\text {sheet }}$ on the step height are summarized in Supplementary Fig. 5, the general trend is that larger distances result in higher conductivities. Moreover, comparing terraces connected by steps with almost identical step height (e.g., Supplementary Fig. 5 black: $507 \mathrm{pm}$ and pink: 500 $\mathrm{pm}$ ), we find a large spread of the sheet resistances: $304 \Omega$ vs. $465 \Omega$ and $165 \Omega$ vs. $294 \Omega$ for the black and pink configuration, respectively (see also Supplementary Fig. 6a-c). Height deviations are found for all temperatures (Fig. 3a, Supplementary Figs. 5 and $6 \mathrm{~d}, \mathrm{e}$ ) and the topographic nature of the observed height deviation in CCT is supported by AFM topographies (Supplementary Fig. 7). Details of the height analysis are given in Supplementary Figs. 8 and 9.

In order to take the atomic-scale structure of the sample into account, we acquired higher resolved CCTs on terraces connected by single and double substrate steps as shown in Fig. 4a and Supplementary Fig. 10b, respectively. On all terraces the $6 \times 6$ quasi corrugation (The wording "quasi" modulation is used, because it consists of two types of ring like structures with slightly different size. One large and two smaller rings together form the superstructure ${ }^{35}$.) is visible. It is induced by a lattice mismatch of the graphene sheet and the substrate and originates from actual height corrugation as well as from electronic contrast ${ }^{35-38}$. However, this $6 \times 6$-quasi corrugation is structurally not perfect (compare Fig. 4a). In order to analyze deviations from a perfect ordering, we disentangle the constant current topographies using Fourier analysis (Supplementary Fig. 11). Applying this type of evaluation for each terrace separately, we disentangle three different contributions to the topographic contrast. Firstly, the $6 \times 6$-quasi corrugation itself, secondly short-range noise and thirdly, long-range spatial modulations, which can be understood as perturbations of the $6 \times 6$-quasi corrugation. The latter contributions are shown in Fig. $4 \mathrm{~b}, \mathrm{c}$ for the terraces to the left 
a

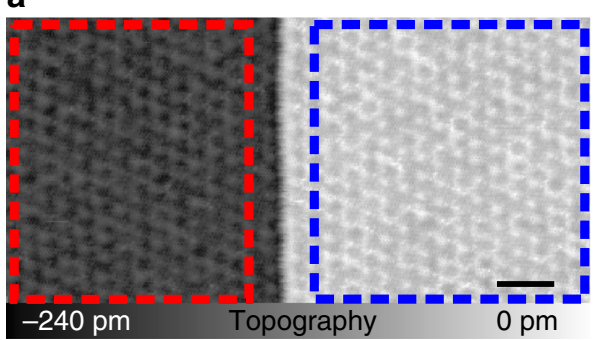

b
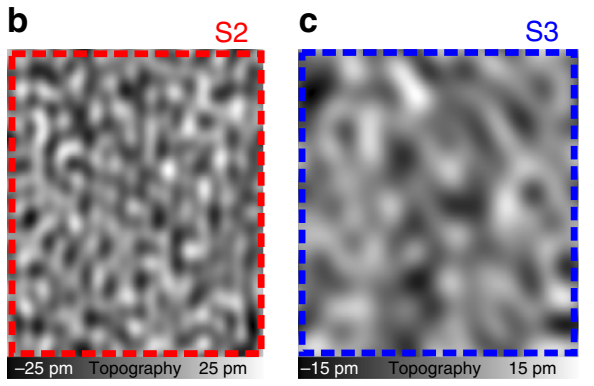

d

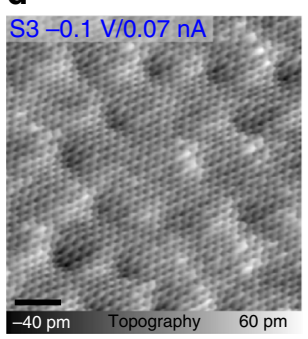

e

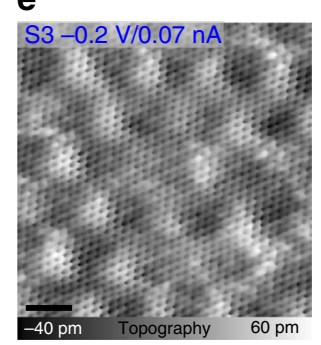

f

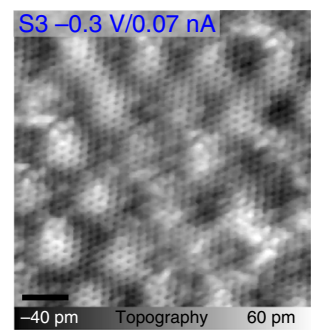



Fig. 4 Analysis of the local defect structure on terraces S2 and S3. a $50 \mathrm{~nm} \times 25 \mathrm{~nm}$ constant current topography of terraces connected by a single substrate step. The scale bar is $5 \mathrm{~nm}$. On both terraces the $6 \times 6$ modulation is well resolved. The topographic contrast is disentangled into its spectral components (as shown in Supplementary Fig. 11) using Fourier analysis. b, c The long-range contributions to the constant current topography are shown for the areas in a marked with dashed red and blue boxes, respectively. d CCT $\left(8 \mathrm{~nm} \times 8 \mathrm{~nm}, I_{\mathrm{T}}=0.07 \mathrm{nA}\right)$ to the left of a double step on a terrace $\mathrm{S} 3$ acquired with a bias voltage of $V_{\text {Bias }}=-0.1 \mathrm{~V}$, e with a bias voltage of $V_{\text {Bias }}=-0.2 \mathrm{~V}$ and $\mathbf{f}$ with a bias voltage of $V_{\text {Bias }}=-0.3 \mathrm{~V}$ representing the integral local density of states in the energy interval $E_{\mathrm{F}} \pm \mathrm{e} V_{\text {bias. }}$. For $\mathbf{d}$, e, $\mathbf{f}$ the scale bar is $1 \mathrm{~nm}$. $\mathbf{g}$ Scanning tunneling spectroscopy at $8 \mathrm{~K}$ on terraces $\mathrm{S} 2$ and $\mathrm{S} 3$ separated by a double substrate step as indicated in the constant current topography in the inset $\left(V_{\mathrm{Bias}}=-0.6 \mathrm{~V}, I_{\mathrm{T}}=0.15 \mathrm{nA}\right)$. The solid blue line shows the averaging of all spectra recorded on S3 and the solid red line shows the averaging of all spectra recorded on S2.

and to the right of the single substrate step in Fig. 4a, respectively. We determine the dominant wavelength of these modulations as shown in Supplementary Fig. 12 and find a clear difference between the two terraces. The terrace to the left of the single substrate step (Fig. 4b) shows a spatial modulation with a shorter wavelength of $4.2 \mathrm{~nm}$ compared with the wavelength of the spatial modulations on the terrace to the right of the single substrate step (Fig. 4c) with $8.1 \mathrm{~nm}$. The corresponding, i.e., reversed finding, holds for terraces connected by a double substrate step (Supplementary Fig. 10b) for which comparable wavelengths of the spatial modulations are extracted. Besides differences in the dominant wavelength, the spatial modulations also exhibit different amplitudes.

In summary, the analysis of the surface morphology allows two conclusions. Firstly, the deviation of the step heights indicates a locally varying distance between the graphene layer and the substrate. Secondly, the $6 \times 6$-quasi corrugation does not show a perfect ordering.

Analysis of the local electronic structure. CCTs taken at different bias voltages have additionally been used to gain insight into the local density of states of the combined graphene/buffer layer/SiC substrate system in a given energy interval $E_{\mathrm{F}} \pm e V_{\text {bias }}$. Since we cannot separate electronic states originating from the buffer layer from states originating from the $\mathrm{SiC}$ substrate, we refer to this part as 'interface layer'. For epitaxial graphene on $\mathrm{SiC}$ it is known that for larger voltages $V_{\text {bias }}$ electronic states of the interface layer become visible in $\mathrm{CCTs}^{39}$. In Fig. $4 \mathrm{~d}-\mathrm{f}$, we show high-resolution, quasi-simultaneous CCTs recorded at $V_{\text {bias }}=-0.1 \mathrm{~V}, V_{\text {bias }}=-0.2 \mathrm{~V}$ and $V_{\text {bias }}=-0.3 \mathrm{~V}$. In all images the graphene honeycomb lattice as well as the $6 \times 6$-quasi corrugation are well resolved. They dominate the topographic contrast at $V_{\text {bias }}=-0.1 \mathrm{~V}$. In contrast, at higher voltages additional states of the interface are visible as non-periodic defect structures, which agrees with published results ${ }^{39,40}$. An example for defects of the SiC substrate is disorder. It has recently been shown by X-ray standing wave analysis ${ }^{41}$ that the top layer of the $\mathrm{SiC}$ substrate is Si depleted. This result is in qualitative agreement with a recent HRTEM study ${ }^{42}$ revealing a gradual depletion of $\mathrm{Si}$ across the topmost three bilayers. The depletion is due to the partial decomposition of the top $\mathrm{SiC}$ layers during the growth process leading to a varying Si concentration. This type of substrate disorder might also be present in PASG graphene.

Spectroscopic measurements using scanning tunneling spectroscopy (STS) at $8 \mathrm{~K}$ provide insight into the local electronic structure. $\mathrm{d} I / \mathrm{d} V$ spectra of graphene on $\mathrm{SiC}$ show two prominent minima, firstly the so-called pseudogap at $0 \mathrm{meV}$ and secondly a minimum at the position of the Dirac point ${ }^{43}$. The position of the latter minimum gives a hint to the local charge carrier density ${ }^{44}$. In Fig. $4 \mathrm{~g}$ the STS data acquired on two terraces connected by a double substrate step are shown. The $\mathrm{d} I / \mathrm{d} V$ spectra in Fig. $4 \mathrm{~g}$ are very similar and in agreement with ARPES measurements (Supplementary Fig. 13) and published results ${ }^{43,45}$. Quantitative deviations between STS and ARPES measurements may be due to different measurement conditions such as the temperature, the addressability of electronic states in the different techniques and due to the presence of the probe itself in STS measurements. In addition to the two prominent minima, we find a pronounced maximum between $-200 \mathrm{mV}$ and $-250 \mathrm{mV}$, which we assign to the interface states observed in CCT.

On closer inspection of the individual $\mathrm{d} I / \mathrm{d} V$ spectra it can be seen that the electronic properties on the two terraces are not identical and even on a given terrace we find local deviations (Fig. 4g). In order to quantify these deviations, we describe each individual $\mathrm{d} I / \mathrm{d} V$ spectrum in the region of the minimum at 
negative voltages with a polynomial fit (Supplementary Fig. 14). From the minima of these fits we obtain the position of the Dirac point for each spectrum separately. The variations on a given terrace regarding the position of the Dirac point are comparable to the differences in $\mathrm{d} I / \mathrm{d} V$ spectra on the two different terraces (Supplementary Fig. 14). For the terrace to the left we find an average value of $E_{\mathrm{D}}=(-360 \pm 17) \mathrm{meV}$, for the terrace to the right the mean value is $E_{\mathrm{D}}=(-355 \pm 13) \mathrm{meV}$. The error interval is the standard deviation.

\section{Discussion}

In order to interpret the local transport properties of PASG graphene, we correlate the structural and electronic STM/STS information with the local STP measurement and thereby address a number of questions. Firstly, can we assign the two distinct sheet resistances $\rho_{\text {Low }}$ and $\rho_{\text {High }}$ unambiguously to characteristics of the sample? Secondly, what causes the huge spread in the sheet resistance at low temperature found for both $\rho_{\text {Low }}$ and $\rho_{\text {High }}$ ? And finally, can both effects, the differences in $\rho_{\text {Low }}$ and $\rho_{\text {High }}$ as well as the spread at low temperature, be traced back to the same origin?

In a first step, we assign the specific step structure revealed in large scale topographies (Fig. 1a) to the stacking sequence of the $6 \mathrm{H}-\mathrm{SiC}(0001)$ substrate. All SiC crystals consist of fundamental layers of silicon and carbon atoms, arranged in tetrahedral coordination ${ }^{46-48}$, referred to as fundamental bilayers. Although the $6 \mathrm{H}-\mathrm{SiC}(0001)$ exhibits six different (crystal) terminations (labeled as S1, S2, S3 and $\mathrm{S}^{*}, \mathrm{~S}^{*}, \mathrm{~S}^{*} 49$ in Supplementary Fig. 15), only four out of the six possible $6 \mathrm{H}-\mathrm{SiC}$ terminations are found ${ }^{28}$, because the terraces $\mathrm{S} 1 / \mathrm{S} 1^{\star}$ have a higher decomposition velocity 34,50 compared with the other terraces and therefore disappear during the growth process. We label the graphene terraces according to the substrate terminations as $\mathrm{S} 2 / \mathrm{S} 2^{\star}$ and $\mathrm{S} 3 / \mathrm{S}^{*}$. It directly follows, that graphene on terraces $\mathrm{S} 2 / \mathrm{S}^{*}$ exhibits a low sheet resistance and a short-wave spatial modulation of the $6 \times 6$-quasi corrugation. In contrast, a larger sheet resistance $\rho_{\text {High }}$ and long-wave perturbations of the $6 \times 6$-quasi corrugation are measured on terraces $\mathrm{S} 3 / \mathrm{S}^{\star}$. A systematic difference in $\rho_{\text {High }}$ for S3 compared with S3 ${ }^{*}$ and in $\rho_{\text {Low }}$ for S2 compared with $\mathrm{S}^{*}$ was not observed (Supplementary Fig. 9). Therefore, we refer to $\mathrm{S} 3 / \mathrm{S} 3^{*}$ as $\mathrm{S} 3$ and $\mathrm{S} 2 / \mathrm{S} 2^{*}$ as S2 in the following (compare Fig. 1c). In summary, we conclude that S2 and S3 are characterized by sheet resistances, which differ by their absolute values as well as by their temperature dependence.

We continue our discussion with a more detailed comparison of the local structural and electronic properties of PASG graphene. In general, a variation in the sheet resistance can be caused by a modified charge carrier density, e.g., in the framework of polarization doping ${ }^{51,52}$, as well as a variation in mobility. STS data allow to estimate a difference in the local doping on adjacent terraces. In order to cause the variation in the sheet resistance of $140 \%$ for the given terraces, the change in the doping level is expected to become visible as a significant shift of the position of the Dirac point in the STS data of terrace S2 compared with terrace S3. Since the mean Dirac energy on terrace S2 compared with terrace $\mathrm{S} 3$ is only shifted by $\approx 5 \mathrm{meV}$ (Fig. $4 \mathrm{~g}$, Supplementary Fig. 14), we discard a locally varying polarization doping 51,52 as the main reason for the observed variation of the sheet resistance. Consequently, the local sheet resistance $\rho$ is predominantly governed by the mobility. The latter is the result of a variety of possible scattering mechanisms like e.g., electron-phonon, electron-electron, or electron-defect interaction, which all could be modified by the local structural and electronic properties of the sample.

To disentangle possible scattering processes in PASG graphene, we first take the measured step heights into account assuming that they reflect the distance of the graphene layer to the substrate and correlate them with the local transport properties of S2 or S3 (see Fig. 3b and Supplementary Fig. 6e). Data sets like the one presented in Fig. $3 \mathrm{~b}$ allow for a comparison of two terraces with the same substrate termination, yet different distances of the graphene to the substrate. They directly show that a larger distance results in a reduced sheet resistance. To further test this hypothesis, we sort the sheet resistances according to the step heights (Supplementary Fig. 5, datasets determined at 8K) and, with a single exception, find a match that larger distances result in a reduced sheet resistance. This finding holds for S2 as well as S3 termination.

While the step height variation is not a priori expected, the observed correlation is not surprising. For epitaxial graphene on $\mathrm{SiC}(0001)$, the buffer layer is partially covalently bonded and thus strongly coupled to the $\mathrm{SiC}$ substrate ${ }^{53}$, whereas the graphene layer is only weakly coupled ${ }^{54}$ by van der Waals interaction. Nevertheless, the electronic properties of epitaxial graphene are known to be strongly influenced by the substrate. Epitaxial graphene shows a strong n-type doping ${ }^{1,35,55}$ from interface states ${ }^{51}$ and a limited charge carrier mobility ${ }^{56}$ due to substrate-induced scattering ${ }^{32,33}$. Already a decoupling of the substrate by intercalation leads to an increase in mobility ${ }^{57}$, suspended/freestanding graphene shows the highest mobility ${ }^{13}$ and a reduced charge carrier density ${ }^{58}$. We suggest that for a larger distance the graphene layer decouples from the substrate resulting in a reduced impact of the defect states of the interface. Thus, these terraces exhibit an increased mobility and a reduced sheet resistance compared with terraces where the graphene layer is closer, i.e., more strongly coupled to the substrate.

Within the proposed model, we now discuss the temperaturedependence of the sheet resistance, i.e., an increasing conductivity with decreasing temperature. In the semi-classical Boltzmann transport, an increase in the conductivity with decreasing temperature is attributed to freezing out of electron-phonon ${ }^{59,60}$ and electron-electron scattering ${ }^{61}$. In addition, potential scattering, screening, and their interplay have to be considered in the discussion. While all these processes depend on the charge carrier density, the electron-electron scattering has been found to be most dominant at high temperatures and low doping ${ }^{62}$. ARPES measurements reveal a high charge carrier concentration of $n \approx 1 \times 10^{13} \mathrm{~cm}^{-2}$ (Supplementary Fig. 13) and moreover, our STS results imply a mainly homogeneous carrier density. Thus, the impact of electron-electron scattering can be assumed to be constant on all terraces, i.e., it cannot explain the experimentally observed spread of $\rho_{\text {sheet }}$.

Since the Fermi wavelength of the electrons roughly corresponds to the wavelength of the potential modulations, we have additionally considered phase-coherent transport phenomena. We predominantly observed classical Lorentz magnetoresistance in macroscopic magnetotransport measurements (Supplementary Fig. 3b), therefore we conclude that weak localization (and phasecoherent transport in general) is only weakly pronounced. Therefore, we do not further consider this effect.

Electron-phonon scattering in graphene on $\mathrm{SiC}$ is governed by the contribution from remote interfacial phonons ${ }^{63,64}$. Since the temperature-dependence of the resistance associated with electron-phonon scattering is consistent with our measurements, we attribute a part of the general temperature-dependence to scattering with substrate phonons. Assuming that electron-phonon scattering causes a monotonous decrease of the sheet resistance with decreasing temperature ${ }^{32,33}$, we estimate the phonon contribution $\rho^{\text {el-phonon }}(T)$ as the difference between the mean sheet resistance at $300 \mathrm{~K}$ and the highest measured values at $8 \mathrm{~K}$ on terraces S3. This estimation yields a phonon contribution of $<100 \Omega$. Besides the general decrease in the sheet resistance, our data show an increase 
in the spread of the individual measurements at low temperature (Fig. 2a) accompanied by a reduction in the sheet resistance of up to $\approx 250 \Omega$ when going from $300 \mathrm{~K}$ to $8 \mathrm{~K}$. Within our model, the spread in the data primarily originates from the dependence of the sheet resistance on the distance $d$ to the substrate. From this, it directly follows that a local modification of the interaction between the graphene sheet and the substrate results in a locally varying mobility. For electron-phonon scattering, one would expect stronger electron-phonon scattering for smaller $d$, which does not agree with the observed behavior (Supplementary Fig. 5). This strongly indicates an additional relevant scattering mechanism besides electron-phonon scattering, explicitly depending on $d$.

Triggered by the observations from CCT, i.e., spatial modulations like the ones observed in Fig. $4 \mathrm{a}-\mathrm{c}$, we propose scattering at local defects and potential fluctuations as the additional scattering mechanism: $\rho(T, d)=\rho^{\mathrm{el}-\text { phonon }}(T)+\rho^{\mathrm{el}-\text { defect }}(T, d)$. The topographic contrast in highly resolved CCT is dominated by the $6 \times$ 6-quasi corrugation. It is randomly perturbed (Fig. 4) and consequently, each terrace is unique with respect to its defect structure. This deviation from the perfect ordering of the $6 \times 6$-quasi corrugation induces a random potential scattering. A temperaturedependent impact of potential scattering on the resistivity has been studied for charged impurities ${ }^{65}$. At low temperatures, the impact of Coulomb scattering at charged impurities is reduced due to localization of electrons and associated screening. We propose that the basics of this concept can be transferred to our system, replacing the charged defects by the non-periodic potential fluctuations of the $6 \times 6$-quasi corrugation (Fig. $4 \mathrm{a}-\mathrm{c}$ ) as well as interface states (Fig. 4d-f). At low temperatures, electrons are trapped in the random potential, thereby screening the spatial modulations of the potential landscape. This results in a weaker potential landscape for the remaining transport electrons, thus contributing to the general reduction in the sheet resistance.

Within the framework of potential scattering it is reasonable that the localization of electrons and associated screening depends on the structural characteristics of the respective terrace, i.e., the exact shape of the random potential landscape. Therefore, the defect structure that is expected to change from terrace to terrace leads to a variation in the potential as well as the screening. This becomes visible as the large spread in the sheet resistance at low temperatures.

Having identified the distance between the graphene and the substrate as an important parameter that controls the sheet resistance in general, the question arises whether this parameter also explains the different sheet resistances of the graphene sheet on terraces S2 compared with terraces S3. Figure 3c shows the difference in the sheet resistance for adjacent terraces S2 and S3 with respect to the experimentally determined step height deviation. It reveals no clear dependence of the variation in the sheet resistance for adjacent terraces S2 and S3 on the height deviation and thus implies that, in addition to the distance dependence of the sheet resistance, intrinsic differences between S2 and S3 exist. Although not providing a comprehensive picture, first indications of these intrinsic differences can be found in the wavelength of the structural modulation (Fig. 4) of S2 and S3, in the local defect structure of the interface layer and by comparing the local $I(V)$ spectra acquired on S2 and S3 that show slight differences in the spectra at e.g., $-300 \mathrm{mV}$.

In summary, the spatial homogeneity of PASG graphene allows for a quantitative analysis of electronic transport on a local scale. We have shown a direct correlation of the structural as well as the electronic transport properties with the substrate. In particular, PASG graphene shows a locally inhomogeneous sheet resistance, which is governed by both the substrate termination of the $\mathrm{SiC}$ and the distance between the graphene layer and the substrate. A locally varying distance to the substrate is accompanied by a variation of the impact of the interface states such that a larger distance leads to a reduced resistance. By analyzing the temperature-dependence of the sheet resistance we have disentangled different scattering mechanisms and have thereby revealed a large inhomogeneity in the sheet resistance at low temperatures associated with the imperfections of the $6 \times 6$-quasi corrugation and localized defects.

Besides the investigation of fundamental processes in the interaction between substrate and graphene, the reported anisotropy could be exploited in further transport experiments. For example, it is interesting to simply rotate the sample by $90^{\circ}$ such that the current is applied parallel to the steps instead of perpendicular. Generally, terraces S2 with $\rho_{\text {low }}$ carry more current than terraces S3 with $\rho_{\text {High }}$ depending on the ratio of the two sheet resistances (see Supplementary Fig. 16) yielding transport channels with a minimum width of about ten times the Fermi wavelength. Thus, by selecting suitable narrow terraces, quasi $1 \mathrm{D}$ electronic transport might be accessible in a $2 \mathrm{D}$ sample. In addition, terraces S2 act as nanoscale heat sources and terraces S3 as heat sinks. This enables the investigation of thermal transport in low dimensions. Thus, PASG graphene can be a model system to study the interplay between electronic and heat transport with the aim of improving the performance of thermoelectric devices $^{66}$. In this context, the question arises as to the limitations of the reported effect, i.e., a maximum variation in the sheet resistance of $270 \%$ at low temperatures. Such a strong local inhomogeneity of the electronic transport is an important quality in the field of epitaxial graphene. It e.g., implies that nanometersized devices could exhibit a local variation in the mobility of up to $270 \%$. We are convinced that the findings of this study can be generalized and should be considered for other 2D materials grown on and in proximity with a substrate.

\section{Methods}

Sample preparation. Graphene samples investigated in this study were grown on the (0001) Si-terminated face of semi-insulating $6 \mathrm{H}-\mathrm{SiC}$ wafers with small nominal miscut angle of $0.06^{\circ}$ toward [1 $\left.1 \overline{100}\right]$ direction applying the PASG technique ${ }^{9,28}$. The idea of this method is to support the growth process with an external carbon source. A polymer is deposited on the substrate using liquid phase deposition before high-temperature sublimation growth is initialized ${ }^{9,10,28}$. The samples were initially annealed in vacuum $\left(p \approx 4 \cdot 10^{-7} \mathrm{mbar}, 900^{\circ} \mathrm{C}, 30 \mathrm{~min}\right)$. The process was proceeded by intermediate annealing in argon ambient $(p=900 \mathrm{mbar})$ at $1200^{\circ} \mathrm{C}$ and $1400^{\circ} \mathrm{C}$ for 10 and $2 \mathrm{~min}$, respectively. Afterward, the samples were heated directly up to $1750{ }^{\circ} \mathrm{C}$ and annealed $(6 \mathrm{~min})$ while argon flux was kept at $0 \mathrm{sccm}^{10}$. Samples prepared with this method are almost defect- and bilayer-free and exhibit shallow step heights, as verified in Raman mapping and AFM topographies 9,28 .

Scanning probe measurements. The experiments were performed in a custombuilt low-temperature STM and in a custom-built room temperature STM under UHV conditions (base pressure $<10^{-10} \mathrm{mbar}$ at $300 \mathrm{~K}, 77 \mathrm{~K}$, and $8 \mathrm{~K}$ ) using electrochemically etched tungsten tips. STS spectra were acquired using standard lockin technique and a modulation amplitude of $10 \mathrm{mV}$. The concept of the STP setup is depicted in Supplementary Fig. 17a. We electrically contact our samples ( $3 \mathrm{~mm} \times 7 \mathrm{~mm}$ ) with gold contacts of $50-100 \mathrm{~nm}$ thickness by thermal evaporation in a shadow mask procedure in a two-terminal geometry. In order to eliminate surface contamination, the samples are heated up to $400^{\circ} \mathrm{C}$ for $30 \mathrm{~min}$ after reinsertion into the UHV chamber. A voltage $V_{\text {cross }}$ is applied across the sample via two gold contacts. The voltage $V_{\mathrm{STP}}(x, y)$, which is a measure of the local electrochemical potential, is adjusted such that the net tunnel current $I_{\mathrm{T}}$ vanishes and is additionally recorded as a function of position. The resulting potential map (Supplementary Fig. 17b) gives access to the voltage drop along the graphene sheet in the investigated sample area. The simultaneously acquired constant current topography (Supplementary Fig. 17c) allows to directly connect transport and topographic information. The local sheet resistance of each terrace is determined from the potential gradient on the terrace and the current density $j$ as follows ${ }^{4}$ $\rho_{\text {sheet }}=\frac{d V_{\text {STP }}}{d x} \cdot \frac{1}{j}=\frac{E_{\mathrm{x}}}{j}$.

Finite element simulation with COMSOL. The local current density $j_{\text {local }}(x, y)$ was calculated using a finite element simulation based on COMSOL multiphysics 
using the AC/DC module. As input parameters, we enter the macroscopic ohmic resistance and the global geometry of the sample. Additional topographic information like substrate steps, bilayer regions, and corresponding monolayer-bilayer transitions are included according to the structural information from constant current topographies. Step resistivities used in this study are set to $6 \Omega \mu \mathrm{m}, 12 \Omega \mu \mathrm{m}$, $18 \Omega \mu \mathrm{m}$ for single, double, and triple substrate steps, respectively.

\section{Data availability}

The authors declare that all data supporting the findings of this study are available within the paper and its supplementary information files. Source data for Figs. 2a, b and $3 \mathrm{c}$ are provided as Source Data file.

Received: 12 July 2019; Accepted: 16 December 2019;

Published online: 28 January 2020

\section{References}

1. Berger, C. et al. Ultrathin epitaxial graphite: 2D electron gas properties and a route toward graphene-based nanoelectronics. J. Phys. Chem. B 108, 19912-19916 (2004).

2. Muralt, P. \& Pohl, D. Scanning tunneling potentiometry. Appl. Phys. Lett. 48, 514-516 (1986).

3. Miccoli, I., Edler, F., Pfnür, H. \& Tegenkamp, C. The 100th anniversary of the four-point probe technique: the role of probe geometries in isotropic and anisotropic systems. J. Phys.: Condens. Matter 27, 223201 (2015).

4. Willke, P., Schneider, M. A. \& Wenderoth, M. Electronic transport properties of 1D-defects in graphene and other 2D-systems. Ann. Phys. 529, 1700003 (2017).

5. Ji, S.-H. et al. Atomic scale transport in epitaxial graphene. Nat. Mat. 11, 114-119 (2011).

6. Willke, P., Druga, T., Ulbrich, R. G., Schneider, M. A. \& Wenderoth, M. Spatial extent of a Landauer residual-resistivity dipole in graphene quantified by scanning tunnelling potentiometry. Nat. Commun. 6, 6399 (2015).

7. Clark, K. W. et al. Spatially resolved mapping of electrical conductivity across individual domain (grain) boundaries in graphene. ACS Nano 7, 7956-7966 (2013).

8. Emtsev, K. V. et al. Towards wafer-size graphene layers by atmospheric pressure graphitization of silicon carbide. Nat. Mat. 8, 203-207 (2009).

9. Kruskopf, M. et al. Comeback of epitaxial graphene for electronics: large-area growth of bilayer-free graphene on SiC. 2D Mater. 3, 041002 (2016).

10. Momeni Pakdehi, D. et al. Homogeneous large-area quasi-free-standing monolayer and bilayer graphene on SiC. ACS Appl. Nano Mater. 2, 844-852 (2019).

11. Dean, C. R. et al. Boron nitride substrates for high-quality graphene electronics. Nat. Nanotech. 5, 722-726 (2010).

12. Riedl, C., Coletti, C., Iwasaki, T., Zakharov, A. A. \& Starke, U. Quasi-freestanding epitaxial graphene on $\mathrm{SiC}$ obtained by hydrogen intercalation. Phys. Rev. Lett. 103, 246804 (2009).

13. Bolotin, K. I. et al. Ultrahigh electron mobility in suspended graphene. Solid State Commun. 146, 351-355 (2008).

14. Ge, J.-L. et al. Weak localization of bismuth cluster-decorated graphene and its spin-orbit interaction. Front. Phys. 12, 127210 (2017)

15. Avsar, A. et al. Spin-orbit proximity effect in graphene. Nat. Commun. 5, 4875 (2014).

16. Song, K. et al. Spin proximity effects in graphene/topological insulator heterostructures. Nano Lett. 18, 2033-2039 (2018).

17. Haugen, H., Huertas-Hernando, D. \& Brataas, A. Spin transport in proximityinduced ferromagnetic graphene. Phys. Rev. B 77, 115406 (2008).

18. Tang, C. et al. Approaching quantum anomalous Hall effect in proximitycoupled YIG/graphene/h-BN sandwich structure. APL Mater. 6, 026401 (2018).

19. Natterer, F. D. Scanning tunneling spectroscopy of proximity superconductivity in epitaxial multilayer graphene. Phys. Rev. B 93, 045406 (2016).

20. Wang, Z.-J. et al. Simultaneous n-interaction and n-doping of epitaxial graphene on $6 \mathrm{H}-\mathrm{SiC}(0001)$ through thermal reactions with ammonia. Nano Res 6, 399-408 (2013).

21. Clark, K. W. et al. Energy gap induced by Friedel oscillations manifested as transport asymmetry at monolayer-bilayer graphene boundaries. Phys. Rev. X 4, 011021 (2014).

22. Willke, P. et al. Local transport measurements in graphene on $\mathrm{SiO}_{2}$ using Kelvin probe force microscopy. Carbon 102, 470-476 (2016).

23. Bipolar gating of epitaxial graphene by intercalation of Ge. Appl. Phys. Lett. 104, 261602 (2014).

24. Baringhaus, J. et al. Ballistic bipolar junctions in chemically gated graphene ribbons. Sci. Rep. 5, 9955 (2015).
25. Giannazzo, F., Deretzis, I., La Magna, A., Roccaforte, F. \& Yakimova, R. Electronic transport at monolayer-bilayer junctions in epitaxial graphene on SiC. Phys. Rev. B 86, 235422 (2012).

26. Ella, L. et al. Simultaneous voltage and current density imaging of flowing electrons in two dimensions. Nat. Nanotech. 14, 480-487 (2019).

27. Druga, T., Wenderoth, M., Homoth, J., Schneider, M. A. \& Ulbrich, R. G. A versatile high resolution scanning tunnelling potentiometry implementation. Rev. Sci. Instrum. 81, 083704 (2010).

28. Momeni Pakdehi, D. et al. Minimum resistance anisotropy of epitaxial graphene on SiC. ACS Appl. Mater. Interfaces 10, 6039-6045 (2018).

29. Landauer, R. Spatial variation of currents and fields due of localized scatterers in metallic conduction. IBM J. Res. Dev. 1, 223-231 (1957).

30. Homoth, J. et al. Electronic transport on the nanoscale: ballistic transmission and Ohm's law. Nano Lett. 9, 1588-1592 (2009).

31. Low, T., Perebeinos, V., Tersoff, J. \& Avouris, P. Deformation and scattering in graphene over substrate steps. Phys. Rev. Lett. 108, 096601 (2012).

32. Jobst, J. et al. Quantum oscillations and quantum Hall effect in epitaxial graphene. Phys. Rev. B 81, 195434 (2010).

33. Jobst, J. et al. Transport properties of high-quality epitaxial graphene on $6 \mathrm{H}-$ SiC(0001). Solid State Commun. 151, 1061-1064 (2011).

34. Yazdi, G. R. et al. Growth of large area monolayer graphene on 3C-SiC and comparison with other SiC polytypes. Carbon 57, 477-484 (2013).

35. Riedl, C., Coletti, C. \& Starke, U. Structural and electronic properties of epitaxial graphene on $\mathrm{SiC}(0001)$. A review of growth, characterization, transfer doping and hydrogen intercalation. J. Phys. D: Appl. Phys. 43, 374009 (2010).

36. Sclauzero, G. \& Pasquarella, A. Stability and charge transfer at the interface between $\mathrm{SiC}(0001)$ and epitaxial graphene. Microelectron. Eng. 88, 1478-1481 (2011).

37. Kim, S., Ihm, J., Choi, H. J. \& Son, Y.-W. Origin of anomalous electronic sturctures of epitaxial graphene on silicon carbide. Phys. Rev. Lett. 100, 176802 (2008).

38. Berger, C., Conrad, E. H., de Heer, W. A., Epigraphene: epitaxial graphene on silicon carbide. in Physics of solid surfaces, Vol III/45B (eds Subvolume, B., Chiarotti G. \& Chiaradia, P.), (Springer-Verlag Berlin Heidelberg, 2017)

39. Rutter, G. M. Imaging the interface of epitaxial graphene with silicon carbide via scanning tunneling microscopy. Phys. Rev. B 76, 235416 (2007).

40. Cervenka, J., van de Ruit, K. \& Flipse, C. F. J. Giant inelastic tunneling in epitaxial graphene mediated by localized states. Phys. Rev. B 81, 205403 (2010).

41. Conrad, M. et al. Structure and evolution of semiconducting buffer graphene grown on $\mathrm{SiC}(0001)$. Phys. Rev. B 96, 195304 (2017).

42. Gruschwitz, M., Schletter, H., Schulze, S., Alexandrou, I. \& Tegenkamp, C. Epitaxial graphene on $6 \mathrm{H}-\mathrm{SiC}(0001)$ : defects in $\mathrm{SiC}$ investigated by STEM. Phys. Rev. Mat. 3, 094004 (2019).

43. Willke, P. et al. Doping of graphene by low-energy ion beam implantation: structural, electronic, and transport properties. Nano Lett. 15, 5110-5115 (2015).

44. Joucken, F. et al. Localized state and charge transfer in nitrogen-doped graphene. Phys. Rev. B: Condens. Matter Mater. Phys. 85, 161408 (2012).

45. Brar, V. W. et al. Scanning tunneling spectroscopy of inhomogeneous electronic structure in monolayer and bilayer graphene on SiC. Appl. Phys. Lett. 91, 122101 (2007).

46. Schardt, J. et al. LEED structure determination of hexagonal $a$-SiC surfaces. Surf. Sci. 337, 232-242 (1995)

47. Park, C. H., Byoung-Ho, C., Keun-Ho, L. \& Chang, K. J. Structural and electronic properties of cubic, $2 \mathrm{H}, 4 \mathrm{H}$, and $6 \mathrm{H} \mathrm{SiC}$. Phys. Rev. B 49, 4485-4492 (1994).

48. Hristu, R., Stanciu, S. G., Tranca, D. E., Polychroniadis, E. K. \& Stanciu, G. A Identification of stacking faults in silicon carbide by polarization-resolved second harmonic generation microscopy. Sci. Rep. 7, 4870 (2017).

49. Seyller, T. Passivation of hexagonal $\mathrm{SiC}$ surfaces by hydrogen termination. $J$. Phys.: Condens. Matter 16, S1755 (2004).

50. Borovikov, V. \& Zangwill, A. Step bunching of vicinal $6 \mathrm{H}-\mathrm{SiC}\{0001\}$ surfaces. Phys. Rev. B 79, 245413 (2009).

51. Ristein, J., Mammadov, S. \& Seyller, T. Origin of doping in quasi-free-standing graphene on silicon carbide. Phys. Rev. Lett. 108, 246104 (2012).

52. Mammadov, S. et al. Polarization doping of graphene on silicon carbide. $2 D$ Mater. 1, 035003 (2014)

53. Emtsev, K. V., Speck, F., Seyller, T. \& Ley, T. Interaction, growth, and ordering of epitaxial graphene on $\mathrm{SiC}\{0001\}$ surfaces: a comparative photoelectron spectroscopy study. Phys. Rev. B 77, 155303 (2008).

54. Varchon, F. et al. Electronic structure of epitaxial graphene layers on SiC: effects of the substrate. Phys. Rev. Lett. 99, 126805 (2007).

55. Bostwick, A., Ohta, T., Seyller, T., Horn, K. \& Rotenberg, E. Quasiparticle dynamics in graphene. Nat. Phys. 3, 36-40 (2007).

56. Pallecchi., E. et al. High electron mobility in epitaxial graphene on $4 \mathrm{H}-\mathrm{SiC}$ (0001) via post-growth annealing under hydrogen. Sci. Rep. 4, 4558 (2014). 
57. Speck, F. et al. The quasi-free-standing nature of graphene on $\mathrm{H}$-saturated $\mathrm{SiC}$ (0001). Appl. Phys. Lett. 99, 122106 (2011).

58. Du, X., Skachko, I., Barker, A. \& Andrei, E. Y. Approaching ballistic transport in suspended graphene. Nat. Nanotech. 3, 491-495 (2008).

59. Hwang, E. H. \& Das Sarma, S. Screening-induced temperaturedependent transport in two-dimensional graphene. Phys. Rev. B 79, 165404 (2009).

60. Das Sarma, S. \& Hwang, E. H. Density-dependent electrical conductivity in suspended graphene: approaching the Dirac point in transport. Phys. Rev. B 87, 035415 (2013).

61. Kotov, V. N., Uchoa, B. \& Pereira, V. M. Electron-electron interactions in graphene: current status and perspectives. Rev. Mod. Phys. 84, 1067-1125 (2012).

62. Mendoza, M., Herrmann, H. J. \& Succi, S. Hydrodynamic model for conductivity in graphene. Sci. Rep. 3, 1052 (2013).

63. Chen, J.-H., Jang, C., Xiao, S., Ishigami, M. \& Fuhrer, M. S. Intrinsic and extrinsic performance limits of graphene devices on $\mathrm{SiO}_{2}$. Nat. Nanotech. 3, 206-209 (2008).

64. Fratini, S. \& Guinea, F. Substrate-limited electron dynamics in graphene. Phys. Rev. B 77, 195415 (2008).

65. Stauber, T., Peres, N. M. R. \& Guinea, F. Electronic transport in graphene: a semiclassical approach including midgap states. Phys. Rev. B 76, 205423 (2007).

66. Cao, B.-Y., Yao, W.-J. \& Ye, Z.-Q. Networked nanoconstrictions: an effective route to tuning the thermal transport properties of graphene. Carbon 96, 711-719 (2016).

\section{Acknowledgements}

A.S. acknowledges financial support from the Deutsche Forschungsgemeinschaft (DFG) through project We 1889/13-1. D.M.P. acknowledges support from the School for Contacts in Nanosystems (NTH nano). P.W. acknowledges support from Institute for Basic Science IBS-R027-D1. We acknowledge support by the Open Access Publication Funds of the Göttingen University and from the Deutsche Forschungsgemeinschaft (DFG) through project TE 386/13-1.

\section{Author contributions}

A.S., P.W., M.W. planned the experiments. D.M.P. and K.P. prepared the samples. A.S. and G.A.T. performed the room temperature STM measurements, A.S. acquired the low-temperature data. A.S. carried out the main part of the data analysis, G.A.T. assisted in the analysis of the room temperature data. A.S. performed the COMSOL calculations.
P.S. and F.S. conducted the ARPES measurements. Interpretation of ARPES results was done by P.S., F.S. and T.S.. A.S. produced the van der Pauw device and performed the macroscopic transport measurements. A.S. and M.W. wrote the manuscript. A.S., G.A.T. D.M.P., P.S., P.W., F.S., T.S., C.T., K.P., H.W.S. and M.W. discussed the results and commented on the manuscript.

\section{Competing interests}

The authors declare no competing interests.

\section{Additional information}

Supplementary information is available for this paper at https://doi.org/10.1038/s41467019-14192-0.

Correspondence and requests for materials should be addressed to A.S. or M.W.

Peer review information Nature Communications thanks the anonymous reviewer(s) for their contribution to the peer review of this work.

Reprints and permission information is available at http://www.nature.com/reprints

Publisher's note Springer Nature remains neutral with regard to jurisdictional claims in published maps and institutional affiliations.

Open Access This article is licensed under a Creative Commons Attribution 4.0 International License, which permits use, sharing, adaptation, distribution and reproduction in any medium or format, as long as you give appropriate credit to the original author(s) and the source, provide a link to the Creative Commons license, and indicate if changes were made. The images or other third party material in this article are included in the article's Creative Commons license, unless indicated otherwise in a credit line to the material. If material is not included in the article's Creative Commons license and your intended use is not permitted by statutory regulation or exceeds the permitted use, you will need to obtain permission directly from the copyright holder. To view a copy of this license, visit http://creativecommons.org/ licenses/by/4.0/

(C) The Author(s) 2020 